\title{
Elucidating the context for implementing nonpharmacologic care for neonatal opioid withdrawal syndrome: a qualitative study of perinatal nurses
}

\author{
Clayton J. Shuman ${ }^{1,2,3^{*}}$, Roxanne Wilson ${ }^{4,5}$, Katherine VanAntwerp ${ }^{1}$, Mikayla Morgan ${ }^{1}$ and Ashley Weber ${ }^{6}$
}

\begin{abstract}
Background: Up to 95\% of neonates exposed to opioids in utero experience neonatal opioid withdrawal syndrome at birth. Nonpharmacologic approaches (e.g., breastfeeding; rooming-in; skin-to-skin care) are evidence-based and should be implemented. These approaches, especially breastfeeding, rely on engagement of the neonates' mothers to help deliver them. However, little is known about the structural and social dynamic context barriers and facilitators to implementing maternal-delivered nonpharmacologic care.
\end{abstract}

Methods: Using a qualitative descriptive design, perinatal nurses from a Midwest United States hospital family birthing center, neonatal intensive care unit, and inpatient pediatric unit were interviewed. These units were involved in caring for mothers and neonates affected by opioid use. Telephone interviews followed a semi-structured interview guide developed for this study, were audio-recorded, and lasted about 30-60 min. Interviews were transcribed verbatim and independently analyzed by five investigators using the constant comparative method. Themes were discussed until reaching consensus and subsequently mapped to a conceptual model adapted for this study.

Results: Twenty-one nurses participated in this study (family birth center, $n=9$; neonatal intensive care, $n=6$; pediatrics, $n=6$ ). Analysis resulted in four major themes: 1) Lack of education and resources provided to staff and mothers; 2) Importance of interdisciplinary and intradisciplinary care coordination; 3) Flexibility in nurse staffing models for neonatal opioid withdrawal syndrome; and 4) Unit architecture and layout affects maternal involvement. Minor themes supported each of the four major themes. All themes mapped to the conceptual model.

Conclusions: This study provides a more comprehensive understanding of the barriers and facilitators affecting implementation of maternal involvement in nonpharmacologic care of newborns with neonatal opioid withdrawal syndrome. Future efforts implementing nonpharmacologic approaches must consider the context factors affecting implementation, including structural and social factors within the units, hospital, and broader community.

Keywords: Neonatal abstinence syndrome, Perinatal substance use, Maternal engagement, Nonpharmacologic care, Neonatal opioid withdrawal syndrome, Implementation

${ }^{*}$ Correspondence: clayshu@med.umich.edu

${ }^{1}$ School of Nursing, University of Michigan, 400 N. Ingalls, Ste. 4162, Ann Arbor, MI, USA

Full list of author information is available at the end of the article

\section{Background}

Opioid use and misuse in the United States continues to affect neonates [1], with one neonate born suffering from opioid withdrawal every 15 min [2]. Up to $95 \%$ of neonates exposed to opioids in utero experience neonatal 
opioid withdrawal syndrome (NOWS) at birth [3]. NOWS begins shortly after birth and results from termination of the mother's opioid supply after the umbilical cord is severed [4]. NOWS symptoms vary in clinical presentation, ranging from minor (e.g., mild tremors, irritability) to more severe (e.g., gastrointestinal distress, seizures) [5]. Common outcomes for neonates diagnosed with NOWS include admission to a neonatal intensive care unit (NICU), receipt of pharmacological treatment (e.g., morphine), prolonged hospitalizations, increased costs and resource utilization, and concurrent birth complications (e.g., jaundice, low birth weight, feeding difficulties) [3]. In addition to adverse clinical outcomes, neonates with NOWS are more likely to be separated from their mothers during their hospital stay and after discharge which can affect maternal-infant bonding, family dynamics, and long-term child health and safety [3-5].

Treatment for NOWS is informed by routine assessment of symptom severity (e.g., Finnegan scoring) and often involves both evidence-based nonpharmacologic as first line treatment (e.g., breastfeeding, skin-to-skin care) and pharmacologic approaches [6]. The Eat, Sleep, Console approach has garnered attention nationally and prioritizes nonpharmacologic interventions before initiating pharmacologic interventions [7]. Nonpharmacological interventions have been shown to decrease infant lengths of stay and need for pharmacologic interventions [8]. However, to achieve these outcomes, these evidencebased practices for NOWS must be implemented in clinical practice [6].

Nonpharmacologic approaches for NOWS treatment include a constellation of interventions such as soothing techniques (e.g., swaddling, pacifier use, low stimulation environment) and nutrition and feeding approaches (e.g., breastfeeding, smaller and more frequent feedings, use of infant formulas formulated for sensitive gastrointestinal systems) [8-13]. Optimal treatment of NOWS includes delivery of rooming-in [8-11], breastfeeding [12, 13], and skin-to-skin contact $[12,13]$; thus the mother must be considered as a primary provider of nonpharmacologic treatment. However, mothers are often not present at the bedsides of neonate's with NOWS, leading to low breastfeeding rates and limited delivery of skin-to-skin care for this population $[4,9,10,12,14,15]$. Failure to implement these approaches are due in part to individual-level barriers such as clinician attitudes and stigma, maternal feelings of guilt, and lack of clinician education on NOWS [15-17].

In additional to individual-level factors, the clinical context may also act as a barrier or facilitator to implementation. However, little is known about how the clinical context (e.g., health system, perinatal unit) affects engagement of mothers in implementation of nonpharmacologic care. Families affected by NOWS have recognized contextual barriers to maternal engagement in care, such as inconsistencies among clinicians and repeated transfers between units (e.g., labor and delivery, NICU, pediatrics) [18]. However, a more comprehensive understanding of the contextual barriers and facilitators to implementing maternal-delivered nonpharmacological care is needed to help hospitals, perinatal units, and community service organizations improve neonatal and maternal outcomes related to NOWS. The purpose of this qualitative exploratory study was to examine perinatal, neonatal, and pediatric nurse's perceptions regarding the contextual barriers and facilitators related to maternal engagement in and delivery of nonpharmacologic care of neonates with NOWS.

\section{Methods \\ Design}

A qualitative descriptive design was used to identify nurse-perceived contextual barriers and facilitators. A semi-structured interview guide was developed and implemented to elicit the perceptions of perinatal, pediatric, and neonatal intensive care nurses caring for opioid-exposed mothers and neonates (see below). The study was approved by the University of Michigan institutional review board and approved by the study site's institutional review board.

\section{Conceptual model}

This study was informed by an adapted conceptual model originally developed by Shuman and colleagues [19]. The clinical context was conceptualized as comprising internal (within the hospital) and external (in the community or society) structural and social dynamic factors (Fig. 1). Internal structural factors are relatively static and include elements such as clinical staffing models, architectural layout and features, unit policies, and educational programs. External structural factors may include the demographics and economics of the surrounding community, public policies, and community resources. Internal social dynamic factors refer to the roles and relationships of individuals and groups within the clinical context, such as the relationships among leadership and staff, physicians and nurses, and nurses and mothers. Social dynamic factors external to the hospital may include relationships among mothers and their families (e.g., significant other, parents), mothers and their public health departments or community providers (e.g., methadone clinics, primary care providers, pediatricians).

\section{Setting and participants}

Data were collected in 2018 from nurses at a regional hospital in Minnesota with over 70 neonates diagnosed 


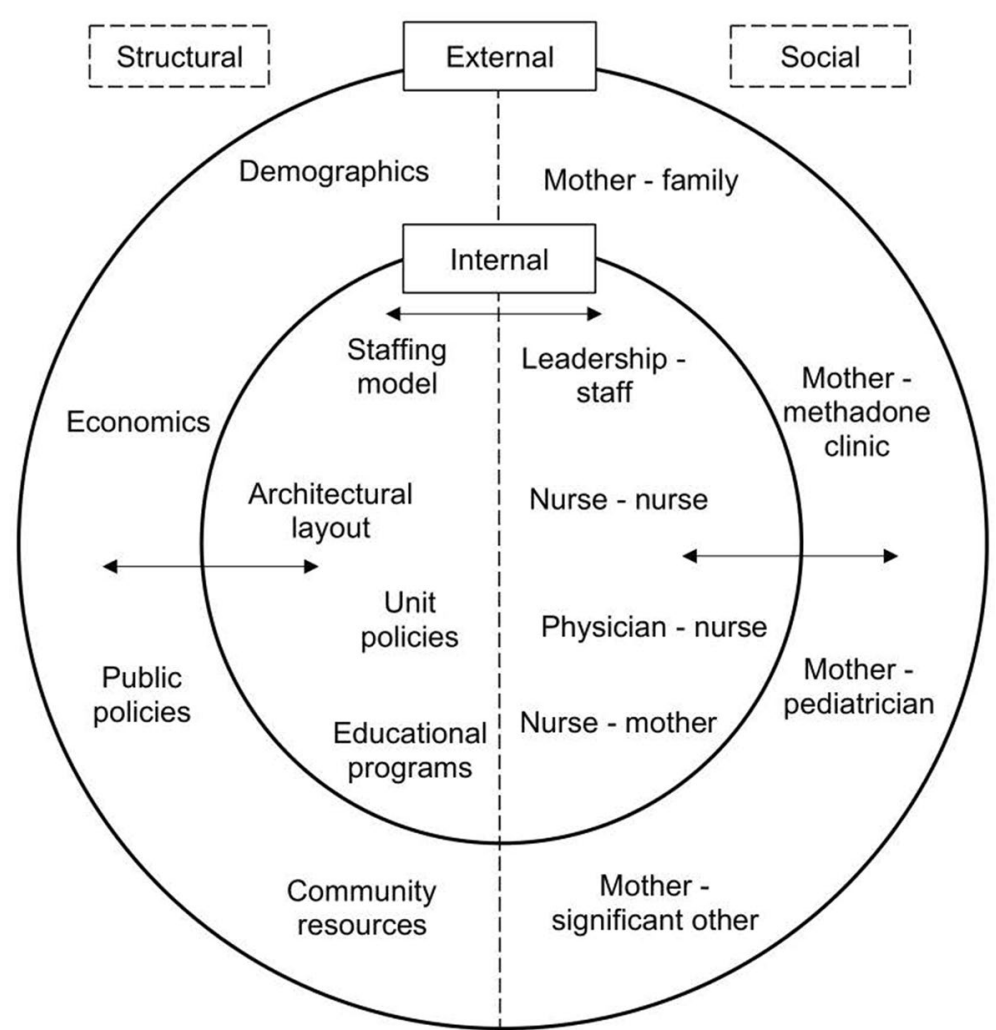

Fig. 1 External and internal social and structural dynamic factors. Legend: Inner circle=internal to the hospital; Outer circle=external to the hospital; Left side of the model = structural factors; Right side of the model=social dynamic factors; Double-sided arrows = interactions between structural and social dynamic factors or across internal and external settings. Dash= relationship between two stakeholders

with NOWS between July 2016 and June 2018. All units caring for opioid-exposed neonates and mothers were included: family birth center (labor and delivery, postpartum and nursery), neonatal intensive care unit (NICU), and inpatient general pediatric unit. Neonates not requiring pharmacologic treatment for NOWS and considered otherwise stable remained at the mothers' bedsides in the family birth center and received care from postpartum nurses trained in NOWS assessment and treatment. Virtual special care was provided to neonates if mothers were eligible for discharge, but their neonates remained on the unit. Virtual special care was not a physical unit and involved monitoring, assessment, and nonpharmacologic treatment while the neonate physically remained in the FBC. Neonates requiring pharmacologic treatment for NOWS were admitted to the pediatric unit. If neonates presented with additional complicating factors (e.g., prematurity), they were admitted to the Level 3 NICU consisting of 30 single patient rooms.

Participants were recruited from these study units who met the following inclusion criteria: 1 ) $\geq 18$ years of age; 2) licensed as a registered nurse; 3 ) employed a minimum of $12 \mathrm{~h}$ per week; 4) designated as staff on a study unit (not float pool or agency); 5) have cared for neonates diagnosed with NOWS in the past 3 years at the study site; and 6) able to speak and understand English.

\section{Procedures}

Eligible participants received an email invitation developed by the research team and sent by the nursing director of each study unit. Those willing to participate in the study enrolled via a web-based form. One member of the study team contacted each participant via telephone to schedule an interview date and time. All interviews were conducted between July and September 2018. Prior to each interview, a study team member reviewed informed consent, answered questions, and obtained verbal consent. A research assistant (KP) and one co-investigator (RW) conducted the interviews independently by telephone. Telephone interviews for qualitative descriptive studies have been shown to be as effective as face-to-face interviews, especially when using semi-structured interview guides [20-23]. Interviews lasted approximately $30-60 \mathrm{~min}$ and were audiorecorded. Participants were offered a \$20 cash card following the interview. 


\section{Instrument}

A semi-structured interview guide was developed and implemented to facilitate interviews and data collection. Topics in the guide were informed by relevant extant literature and external and internal contextual constructs identified in the Shuman and colleagues conceptual model [19] adapted for this study and those included in the Consolidated Framework for Implementation Research (CFIR) model [24]. The guide included introductory and closing scripts, open-ended questions, and probes (see Supplemental Content). Questions and probes were designed to elicit and describe nurse perceptions of contextual factors affecting engagement of mothers in the care of substance-exposed neonates. Although the interview guide was not specific to opioids, all participants primarily focused on opioid withdrawal. This was not surprising because opioid-exposure is most often associated with neonatal withdrawal symptoms [3]. Additional questions in the guide were used to describe individual level (e.g., nurse, mother, neonate) barriers and facilitators, which have been described previously [24]. The guide was rigorously reviewed by the investigative team and stakeholders at the study site (e.g., unit nursing directors, clinical nurse specialists, medical directors) for relevance and comprehensiveness. The flow and content of the guide was tested with two nursing students with clinical and research experience in maternal-infant health.

\section{Data analysis}

Interviews were transcribed verbatim and reviewed for transcription accuracy by comparing transcribed files with audio files. Using the constant comparative methods of Glaser and Strauss [25, 26], all members of the investigative team individually performed initial coding (e.g., minor themes); the team then compared and discussed codes until reaching agreement. Investigators independently organized minor themes into major themes; themes were then compared and discussed until consensus was reached. Qualitative rigor was demonstrated by establishing trustworthiness [25] through assuring credibility (peer debriefing, member-checking, and prolonged engagement), dependability (inquiry audit), and confirmability (reflexivity) [25, 26]. After identifying themes, investigators mapped them to the conceptual model and discussed them until achieving unanimity.

\section{Results}

\section{Participant demographics}

A total of 34 nurse participants who met inclusion criteria enrolled in this study (Table 1). Thirteen of the enrollees were unable to be reached via telephone or email or were unable to schedule an interview date and time. Thus, 21 nurses participated in individual, semistructured interviews. Nine nurses were from the family birth center, six were from the NICU, and six were from the pediatric unit. Participants were mostly female (95\%), Caucasian (100\%), and had a mean age of 37 years $(\mathrm{SD}=10)$.

\section{Themes}

Four major themes were identified: 1) Lack of education and resources provided to staff and mothers; 2) Importance of interdisciplinary and intradisciplinary care coordination; 3) Flexibility in nurse staffing models for NOWS; and 4) Unit architecture and layout affects maternal involvement. Minor themes supported each of the four major themes (Table 2). To demonstrate application of these themes to future research, clinical practice, and public policy, each theme was mapped to our conceptual model (Fig. 2).

\section{Lack of education and resources provided to staff and mothers}

Nurses described a lack of comprehensive unit- or organization-provided NOWS and opioid use disorder (OUD)

Table 1 Participant Demographics $(N=21)$

\begin{tabular}{ll}
\hline & N (\%) \\
\hline Sex & \\
Female & $20(95 \%)$ \\
Highest Nurse Educational Level & \\
Associates & $1(5 \%)$ \\
Bachelors & $18(86 \%)$ \\
Masters & $2(9 \%)$ \\
Advanced Practice Nurse & \\
Yes & $1(5 \%)$ \\
No & $20(95 \%)$ \\
Years of Experience as a Registered Nurse & \\
0-2years & $4(19 \%)$ \\
3-5years & $4(19 \%)$ \\
6-10years & $4(19 \%)$ \\
> 10years & $9(43 \%)$ \\
Years Employed as a Registered Nurse at the Study Hospital \\
0-2years & $5(24 \%)$ \\
3-5years & $5(24 \%)$ \\
6-10years & $3(14 \%)$ \\
> 10years & $8(38 \%)$ \\
Years Employed as a Registered Nurse by their Current Unit \\
0-2years \\
3-5years \\
6-10years & $5(25 \%)$ \\
> 10years & $4(19 \%)$ \\
\hline
\end{tabular}




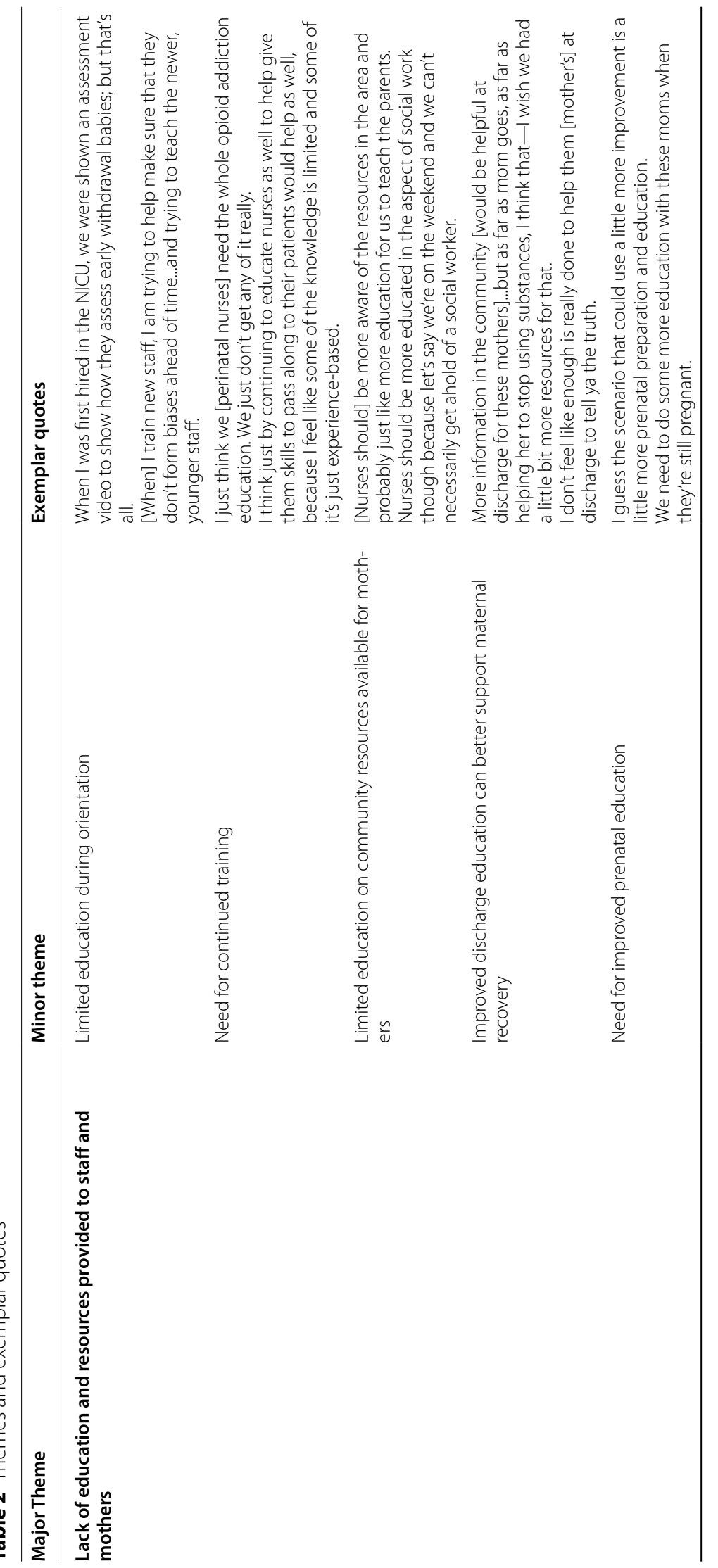




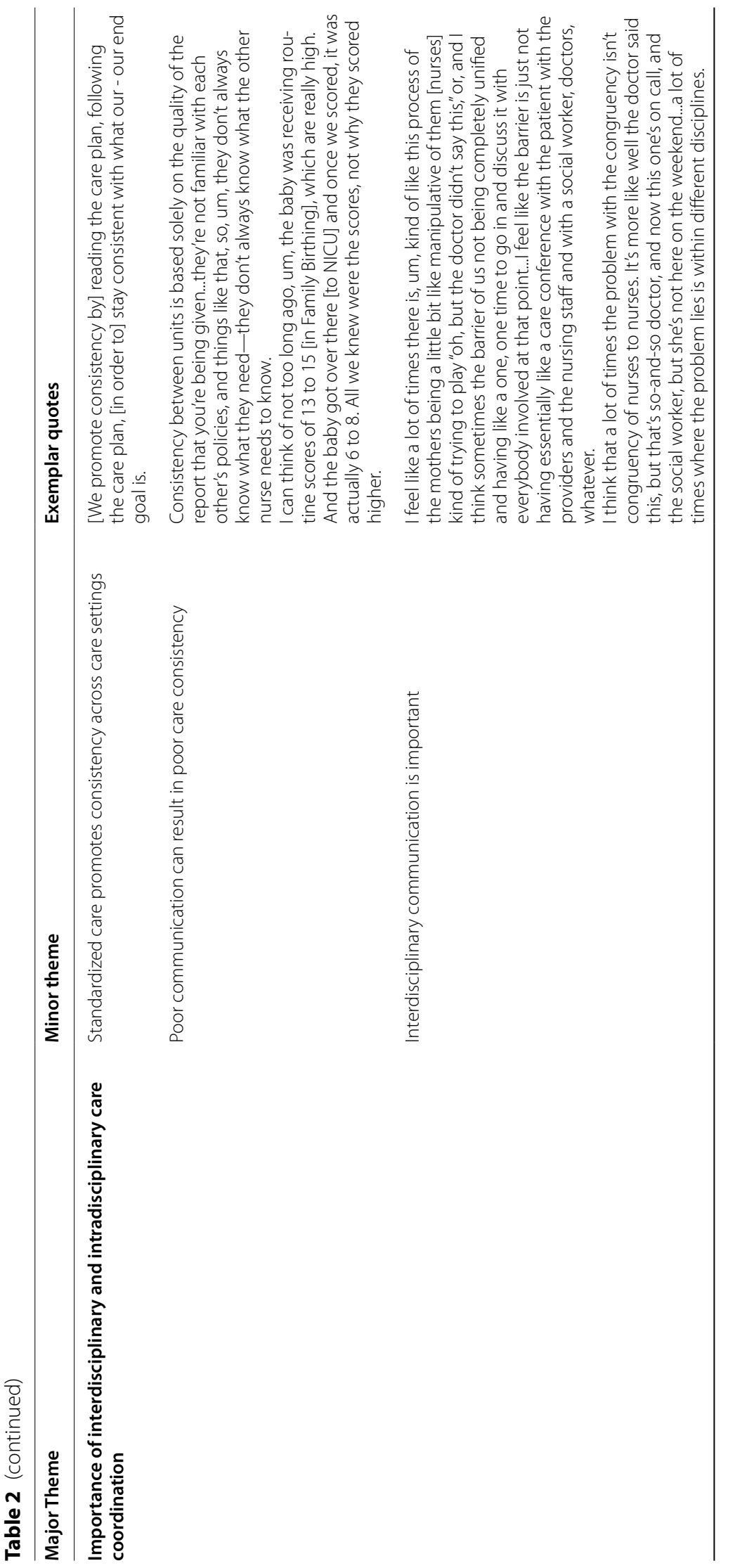




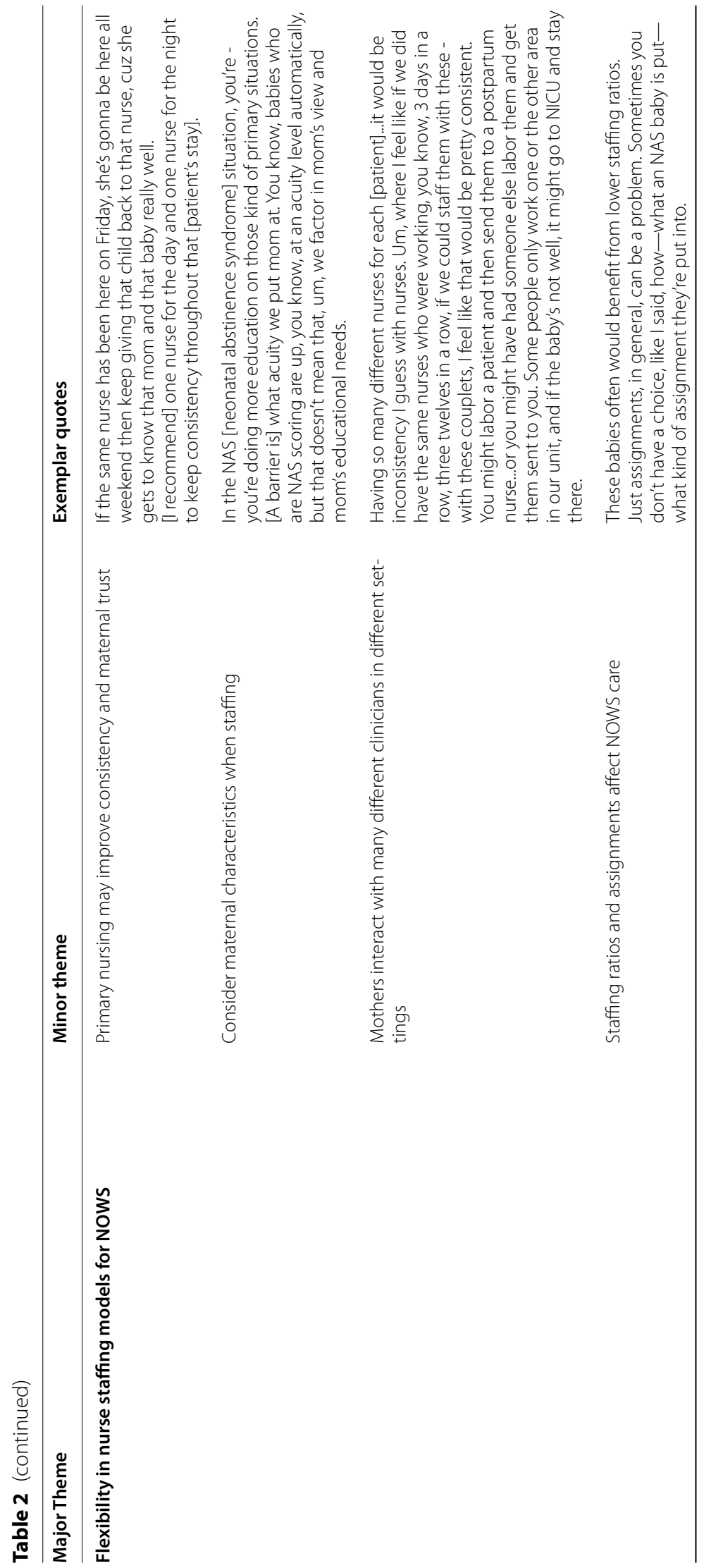




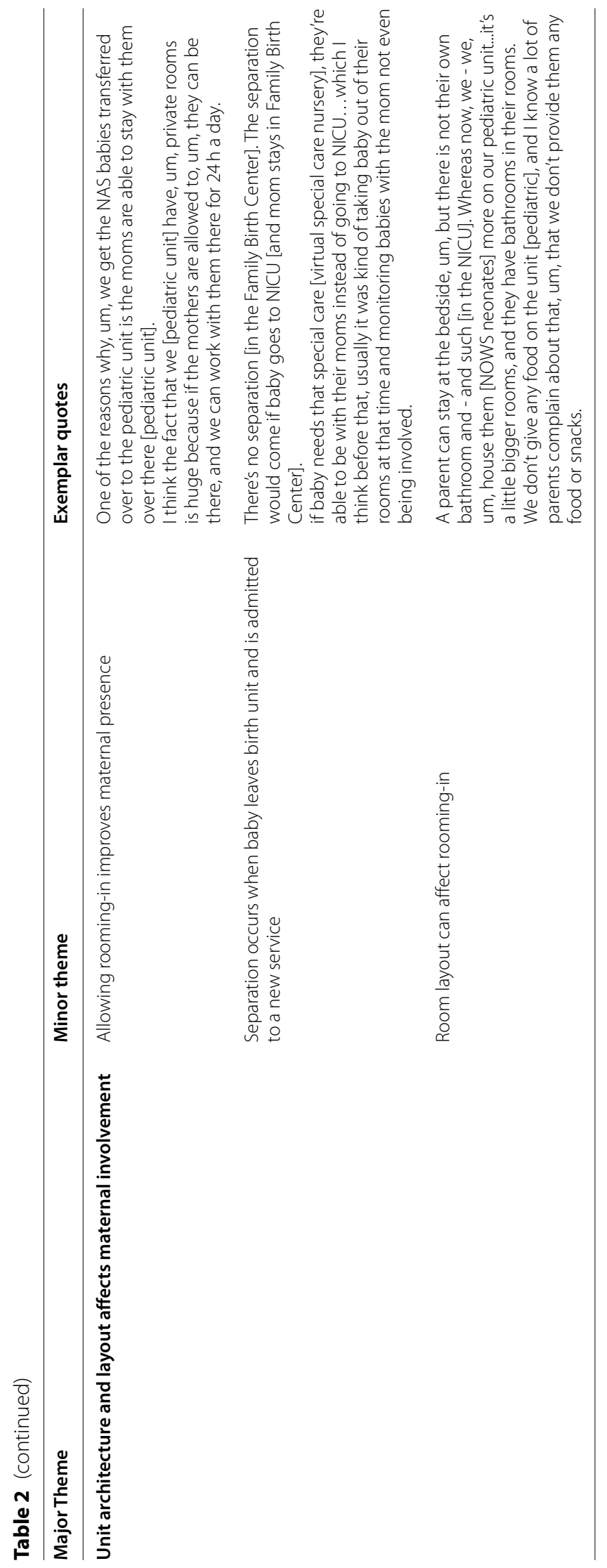




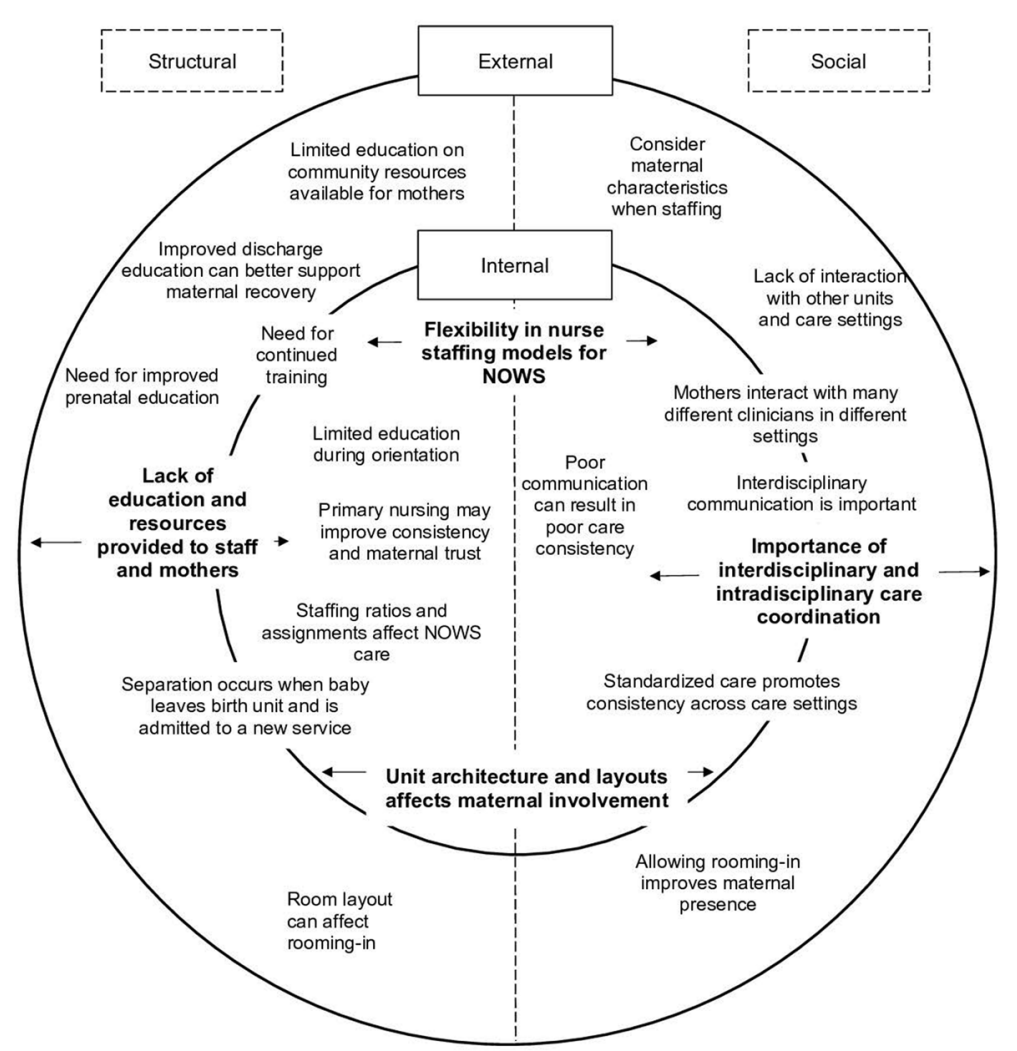

Fig. 2 Themes mapped to the conceptual model. Legend: Bold font= major theme; Normal font=minor theme; Left side of model=structural factors; Right side of model = social dynamic factors; Inner circle = factors internal to the hospital; Outer circle $=$ factors external to the hospital. Double-sided arrows $=$ theme includes structural and social factors or spans internal and external settings

education to effectively care for this patient population. Several nurses recalled NOWS education received during new-hire orientation, but noted it was exclusive to NOWS scoring assessment skills (e.g., Finnegan scoring) without attention to maternal OUD and evidence-based NOWS treatment approaches. Experienced nurses feared new hires did not receive adequate education on how to care for neonates with NOWS, especially concerning the trajectory of maternal OUD and treatment, and the numerous, interacting complex social and family dynamics (e.g., marital status; family proximity; societal stigma). Since hospital provided education was considered lacking, nurses relied on various internet resources (e.g., Google) to learn more about how to care for families affected by NOWS but noted internet resources could be unreliable. Many nurses vocalized experience working with families affected by NOWS; however, they felt their previous experiences were not sufficient to prepare them to care for these maternal-infant dyads. They stated more formalized education from the hospital surrounding maternal OUD (e.g., pathophysiology of addiction; psychology of addiction; trajectory of recovery), NOWS (e.g., long-term infant health and social outcomes; evidence-based treatment approaches), and engaging mothers in treatment of NOWS (e.g., therapeutic communication with mothers with OUD; understanding adverse childhood experiences and trauma) would supplement their experiences and increase their ability to provide quality care to neonates and families affected by NOWS. One nurse summarized, "I just think we [perinatal nurses] need the whole opioid addiction education... we just don't get any of it really."

In addition to nursing education, participants stressed the need for hospitals and communities to enhance the education mothers receive regarding NOWS. A lack in maternal knowledge about NOWS was thought to negatively affect a mother's willingness to engage in neonatal care and implement nonpharmacologic interventions. Several nurses proposed increasing prenatal education about NOWS in community and primary care settings to better prepare mothers for what to expect after delivery. This education could be delivered during prenatal care visits, addiction treatment clinic visits, or through community outreach initiatives. However, nurses acknowledged that many mothers do not receive prenatal care and suggested education provided by labor nurses could 
help set the stage for the entire admission. At discharge, nurses felt mothers were not adequately prepared for a successful transition to home. Specifically, nurses reported mothers were not provided adequate opioid cessation resources at discharge and/or unit and organizational collaboration with community resources and treatment centers were unknown or not well-articulated. Nurses wanted to help mothers and neonates with transition from the hospital to home, but they did not fully understand the trajectory of these patients during and following transitions back to the community.

Nurses in the NICU and pediatric unit emphasized a lack unit and hospital-provided nursing education about challenges faced by mothers with OUD and what resources may be available to help them (e.g., transportation, housing, home visits). Since mothers are not admitted to pediatric or neonatal units, some of these nurses considered the family as "the patient" but felt a strong sense of protection for the neonate who is vulnerable as the "primary patient". In addition, they felt a sense of vulnerability in trying to engage the mother while documenting her actions. This was particularly challenging because nurses understood their charting and interactions could impact findings of Child Protection Services (CPS), yet they had little-to-no interaction with CPS to explain or discuss their concerns.

Many nurses relied on social workers and case managers to connect mothers with community resources (e.g., breastfeeding support, local and state resources, case management, residential treatment, childcare). However, nurses working night or weekend shifts felt less knowledgeable about the availability of hospital and community resources because social workers and case managers were less accessible. Nurses recommended increasing hospital-provided education and collaboration with social work and case management across shifts and in the continuum of care. This would involve considering innovative solutions to utilize social work and case management staff and resources on nights and weekends, when most mothers visited the NICU or pediatric units or when some were discharged.

Nurses identified increased hospital-provided education on addiction, recovery, and post discharge support is needed to improve their practice in interacting with the parents during the neonate's stay and in planning for transition to the community and post discharge care. Without hospital-provided education, nurses described feeling incompetent and unconfident in communicating with mothers with OUD. They felt additional on-the-job training and education could significantly improve their understanding of the mothers' addiction and recovery experiences, leading to increased confidence in engaging these mothers in neonatal care. On the conceptual model, this theme mapped to structural context factors both internal (e.g., hospital-provided education) and external (e.g., community resources for pregnant women with OUD) to the hospital.

\section{Importance of interdisciplinary and intradisciplinary care coordination}

Care coordination was identified as a significant factor contributing to maternal engagement in implementation of nonpharmacologic care and relied on communication across settings (e.g., prenatal, hospital, home/community), interdisciplinary communication, or communication between different providers (e.g., nurses, physicians, social workers), and intradisciplinary communication, or communication within one set of providers (e.g., nurses). Interdisciplinary communication, as described by the nurses from all types of shifts, was compromised between members of the care team (e.g., physician, social work, case manager, lactation consultant, nurse). For example, one nurse stated, "I think that a lot of times the problem with the congruency isn't congruency of nurses to nurses. It's more like well the doctor said this, but that's soand-so doctor, and now this one's on call, and the social worker, but she's not here on the weekend.... lot of times where the problem lies is within different disciplines."

Nurses explained a lack of opportunity to comprehensively discuss care among disciplines contributed to inconsistent care coordination and transition planning (e.g., labor unit to NICU; discharge). One nurse said, "I don't know what social work does once they're out of our hands." Although interdisciplinary care rounds facilitated discussion across disciplines, nurses accentuated a lack of communication regarding NOWS treatment which ultimately led to incongruencies in care and poorer outcomes. Further, mothers were often not involved in planning: "I think that there's just a lot of discussion not in front of the patient [mother] as that transition [to community] and to make that work and when it's appropriate and when it's not."

While describing intradisciplinary communication and care coordination, nurses cited handoff report, nursing notes, bedside report, electronic medical record charting, and nursing care plans as communication modalities contributing to congruent patient care. However, they elaborated and stated the quality of handoff varies considerably across units and between nurses. The variation in the quality of handoff report or communication between units and nurses was described as a leading cause of decreased care quality and continuity. This was thought to adversely affect implementation of maternally provided nonpharmacologic care. For example, nurses suggested handoff reports should include discussion of 
effective and ineffective strategies used by the previous nurse to engage the mother.

Lack of interaction or connectedness between nurses from different units (e.g., labor and NICU) and care settings (e.g., hospital to community), was considered a critical barrier to care coordination, especially to continuation of breastfeeding and skin-to-skin care initiated at delivery. For example, breastfeeding support in a postpartum unit may not have been continued in the NICU when mothers were visiting neonates. In addition to inconsistencies in maternal engagement, nurses reported significant variation in how each unit scored neonates exhibiting symptoms of NOWS. As one nurse explained, labor nurses often scored neonates with suspected NOWS higher than the NICU nurses. Thus, nurses suggested implementing NOWS care practices across all units simultaneously to improve continuity and include community partners in interdisciplinary discharge planning. Standardized care across units and coordination across settings were described as effective strategies to improve engagement of mothers in neonatal care. These strategies were thought to improve maternal engagement by decreasing the level of care disruption mothers and neonates experience as they transition across shifts, units, and hospital and community settings. On the conceptual model, the theme of interdisciplinary and intradisciplinary care coordination mapped to social dynamic context factors, including communication among team members within the hospital (internal) and outside the hospital in the community (external).

\section{Flexibility in nurse staffing models for NOWS}

Nurses described staffing as a major unit and organizational factor contributing to the overall care of neonates with NOWS. Nurses, especially those practicing in the NICU, emphasized primary care nursing, a nursing model that assigns one nurse as a patient's primary care provider for the duration of their hospitalization. Nurses noted primary care nursing, specifically with infants affected by NOWS, can improve care continuity and outcomes by affording increased opportunities for nurses to develop relationships the neonate's family.

Similarly, FBC nurses also emphasized the importance of nursing consistency considering that a mother might labor with one nurse and postpartum with another. Nurses advocated for a more comprehensive approach to NOWS care - an approach that includes all hospital units (e.g., FBC, pediatrics, NICU) and community partners (e.g., treatment clinics, pediatricians, public health nurses) involved in the care of substance-exposed maternal-infant dyads. Episodic care, or unit-based care, can be overly distinct and specific to each unit. This could interfere with continuity of care, especially as nurses prepare mothers and neonates for transition from hospital into the community.

Nurses described neonates with NOWS and their families as having increased needs, both physical and educational, and these needs are often not prominent in determining nurse-patient assignments. As one participant stated, "If they [nurse] have a really heavy assignment and they have a baby who's needing extra care or a mom who needs extra education regarding NAS [neonatal abstinence syndrome] scoring and they're not able to be in there doing that...that is a barrier." Because families affected by NOWS require increased education and social work resources and referrals, nurses felt these patients were of much higher acuity, not accounted for in current acuity indices or patient assignments. When discussing acuity levels and staffing, one nurse said, "[we don't] factor in mom's view and mom's education needs." Accounting for the heightened psychosocial and educational needs of mothers with OUD while making staffing decisions was thought to provide more time for nurses to effectively educate and engage mothers in neonatal care. This theme mapped to internal structural (staffing models) and social dynamic (nurse-mother relationship) context factors in the conceptual model.

\section{Unit architecture and layout affects maternal involvement}

The nurses felt the structural layout of the unit may facilitate or hinder maternal visitation and engagement. Many nurses described the ability for mothers to roomin with their neonate as a facilitator towards maternal involvement. Nurses recognized rooming-in contributed to increased maternal engagement in implementation of nonpharmacologic neonatal care such as breastfeeding and skin-to-skin care and provided numerous opportunities to educate the mother and connect her with community resources. Specifically, nurses described 24-h visitation policies and private rooms as two synergistic factors increasing a mother's intention and ability to room-in with her neonate. However, other factors led to mothers having to leave the room including to go to the restroom, acquiring food, attending individual or group treatment sessions, obtaining methadone. Thus, nurses suggested utilizing rooms with bathrooms, delivering inroom meals for mothers, and providing OUD treatment, including methadone, using in-hospital resources until neonatal discharge.

Nurses from the labor and postpartum units emphasized the importance of the special care nursery in increasing maternal involvement. Nurses described a special care nursery as a virtual unit (not physical unit) which admits neonates with minor NOWS symptoms for monitoring. In the special care nursery, even after maternal discharge the mother had access to a full size bed 
and private bathroom in the neonate's room. Nurses felt this model allowed for increased maternal engagement in neonatal care because the mother and neonate could remain in the same room while the mother recovers from delivery. Further, if required, the neonate would remain in the FBC as a "virtual special care" admission after the mother's discharge. In addition, nurses described the pediatric unit as a prime location for neonatal treatment and they "encouraged moms to go with their babies to pediatrics" if the mother was discharged. Nurses felt that engagement of mothers in neonatal care was impacted by the type of unit where neonates received NOWS treatment, including the ability to room-in. On the conceptual model, this theme mapped to internal structural (e.g., unit architecture) and social dynamic (e.g., improved maternal presence at neonates' bedsides) context factors.

\section{Discussion}

Using a sample of perinatal, pediatric, and neonatal nurses, this study ascertained important contextual factors to consider when implementing nonpharmacologic care for NOWS, both within an acute care setting and in the broader community. The results of this study are timely because NOWS is a pressing public health crisis in the United States [1-3].

These findings compliment growing literature on the perceptions of clinicians and families affected by NOWS $[4,8,10,13,27]$. Previous studies identified individuallevel barriers and facilitators to providing quality care and treatment for NOWS, as related to the clinician (e.g., nurse) or the mother. A common theme across many qualitative studies is the lack of clinician education regarding perinatal opioid use and NOWS. Results of this study support previous findings that perinatal units and healthcare organizations should identify and provide appropriate NOWS training and resources to nurses as well as families. Failure to provide education may result in 1) untrained clinical staff and ill-prepared mothers and 2) significant variation in clinician and maternal education resulting in poorer outcomes and inconsistent care.

Education of staff, although critical, is not sufficient to improve implementation and sustainability of evidence-based practices [28, 29]. Resources, care coordination, and care practices (e.g., staffing models) are also needed to support implementation. For example, Shuman and colleagues (2020) found that limited hospital- or community-based resources (e.g., lack transportation, financial assistance, childcare) often resulted in socioeconomic barriers that prevented mothers from being physically present at the bedside [15]. In the current study, resources promoting consistent care delivery practices and communication across perinatal units within a hospital were found to be essential to promoting breastfeeding, skin-to-skin care, and rooming-in among maternal-infant dyads affected by perinatal opioid use. It is important clinicians understand trajectories of these dyads, including clinical care and resources provided within the hospital, as well as outside the hospital in community-based settings. As argued by Spehr and colleagues, primary care providers are responsible for continuing to support maternally-provided care after discharge from the hospital [27]. Therefore, communication and knowledge regarding perinatal OUD and NOWS are important for hospital-based clinicians as well as community-based clinicians.

Families affected by NOWS often require more support and time from nurses [7]. Managing withdrawal symptoms involves constant monitoring and provision of nonpharmacologic (e.g., holding, pacifier) and sometimes requires pharmacologic interventions $[4,6]$. Nurses with heavier assignments may not have time to provide this care or may not be able to fully care for other patients in their assignment effectively. Improving maternal involvement in neonatal care may reduce some of this workload for bedside nurses and improve patient care. However, it requires nurses to spend significant time training and preparing families to manage NOWS symptoms confidently. This attention, especially during the first few days following birth or diagnosis of NOWS, is critically important to effectively engaging these mothers in neonatal care which may ultimately lead to improved outcomes.

Notably, nurses described the physical layout and architecture of their units as important to engagement of mothers with OUD in the care of their neonates, especially private rooms with private bathrooms. Previous studies have demonstrated that neonates who room-in with their mothers have better outcomes [8-10]. However, for neonates cared for in NICUs, rooming-in may be less frequent depending on unit layout. Generally, the physical layout of NICUs in the United States are either a pod layout (e.g., many infant beds in a room) or offer single family private rooms (e.g., only one infant per room) [30-32]. Pod-style NICUs do not provide private space for mothers to deliver nonpharmacologic care such as breastfeeding or skin-to-skin [31-33]. Further, pods often do not offer a welcoming or conducive environment for 24/7 visitation [22-34]. Private room NICUs are costly and may not be a realistic solution for many hospitals [30, 31]. Even NICUs utilizing single room layouts can challenge rooming-in. For example, in this study, the NICU was structured as single family rooms with only neonatal beds and a couch for visitors, but no private bathrooms or adult beds. Consequently, more efforts may be warranted to identify other units (e.g., pediatric unit) for neonates with NOWS that provide a better environment for rooming-in. In addition to the physical 
capacity to offer rooming-in, health systems are encouraged to consider additional infrastructure supports to encourage mothers to make use of rooming-in, such as delivered meals and treatment support (e.g., methadone dosing; support groups).

To the authors' knowledge, this qualitative study is one of first to describe the contextual challenges of implementing evidence-based care for NOWS treatment, the findings have limitations. First, participants were sampled from a single hospital setting and included nurses only. In addition, participants were mostly female and Caucasian. Participants may have participated in the study because they felt passionately (positively or negatively) about caring for mother and neonates affected by NOWS which may have impacted results. Consequently, it is important to consider replicating this study at other hospitals and with other types of healthcare professionals.

\section{Conclusions}

This study provides a more comprehensive understanding of the barriers and facilitators that affect implementation of maternal involvement in nonpharmacological care of neonates with NOWS. As maternal involvement is essential to the care of neonates with NOWS results in improved outcomes, it is critical to recognize and understand how the clinical and community context can help or impede implementation of evidence-based care. This study identified important contextual factors relevant for NOWS care including the importance of education for staff and mothers, the role of interdisciplinary and intradisciplinary communication across the continuum of care, the need for flexible nurse staffing models, design of pathways of care pre and post discharge, and considerations regarding how the unit architecture and influence outcomes. Future implementation efforts, especially those interested in implementing nonpharmacologic approaches (e.g., Eat, Sleep, and Console), must consider the context factors and individual factors affecting implementation.

\author{
Abbreviations \\ NOWS: Neonatal opioid withdrawal syndrome; NAS: Neonatal abstinence \\ syndrome; OUD: Opioid use disorder; NICU: Neonatal intensive care unit; \\ CFIR: Consolidated framework for implementation research; FBC: Family birth \\ center.
}

\section{Supplementary Information}

The online version contains supplementary material available at https://doi. org/10.1186/s12887-021-02955-y.

Additional file 1.

Acknowledgements

Not applicable.

\section{Authors' contributions}

CS conceptualized, designed, and implemented this study. RW and KV performed individual telephone interviews of nurse participants. CS, MM, RW, AW, and $\mathrm{KV}$ analyzed the results, coded the data, and conceptualized initial major and minor themes. MM and CS drafted the initial manuscript. All authors read and approved the final manuscript.

\section{Funding}

AW is funded through a National Institutes of Health K23 training grant through the National Institute of Nursing Research.

\section{Availability of data and materials}

The datasets used and/or analyzed during the current study are available from the corresponding author on reasonable request.

\section{Declarations}

Ethics approval and consent to participate

This study was approved by the University of Michigan institutional review board and approved by the study site's institutional review board. All methods were carried out in accordance with relevant guidelines and regulations. The study did not involve any experiments. All participants provided informed consent prior to being interviewed.

\section{Consent for publication}

Not applicable.

\section{Competing interests}

The authors declare that they have no competing interests.

\section{Author details}

${ }^{1}$ School of Nursing, University of Michigan, 400 N. Ingalls, Ste. 4162, Ann Arbor, MI, USA. ${ }^{2}$ Institute for Healthcare Policy and Innovation, University of Michigan, Ann Arbor, MI, USA. ${ }^{3}$ Center for the Study of Drugs, Alcohol, Smoking, and Health, University of Michigan, Ann Arbor, MI, USA. ${ }^{4}$ Department of Nursing, St. Cloud State University, St. Cloud, MN, USA. ${ }^{5}$ St. Cloud Hospital, St. Cloud, MN, USA. ${ }^{6}$ College of Nursing, University of Cincinnati, Cincinnati, $\mathrm{OH}, \mathrm{USA}$.

Received: 26 March 2021 Accepted: 15 October 2021

Published online: 04 November 2021

\section{References}

1. Centers for Disease Control and Prevention. Understanding the Epidemic. 2020. https://www.cdc.gov/drugoverdose/epidemic/index.html. Accessed 30 July 2020.

2. Tolia VN, Patrick SW, Bennett MM, Murthy K, Sousa J, Smith B, et al. Increasing incidence of the neonatal abstinence syndrome in U.S. neonatal ICUs. N Engl J Med. 2015;372(22):2118-26. https://doi.org/10.1056/ NEJMsa1500439.

3. McQueen K, Murphy-Oikonen J. Neonatal abstinence syndrome. N Engl J Med. 2016;375:2468-79. https://doi.org/10.1056/NEJMra1600879.

4. Ryan G, Dooley J, Finn LG, Kelly L. Nonpharmacological management of neonatal abstinence syndrome: a review of the literature. J Matern Fetal Neonatal Med. 2019;32(10):1735-40. https://doi.org/10.1080/14767058. 2017.1414180.

5. Finnegan $L P$, Hagan $T$, Kaltenbach KA. Scientific foundation of clinical practice: opiate use in pregnant women. Bull NY Acad Med. 1991;67(3):223-39.

6. Raffaeli G, Cavallaro G, Allegaert K, Wildschut EED, Fumagalli M, Agosti M, et al. Neonatal abstinence syndrome: update on diagnostic and therapeutic strategies. Pharmacotherapy. 2017;37(7):814-23. https://doi.org/ 10.1002/phar.1954.

7. Grisham LM, Stephen MM, Coykendall MR, Kane MF, Maurer JA, Bader MY. Eat, sleep, console approach: a family-centered model for the treatment of neonatal abstinence syndrome. Adv Neonatal Care. 2019;19(2):138-44. https://doi.org/10.1097/ANC.0000000000000581. 
8. Holmes AV, Atwood EC, Whalen B, et al. Rooming-in to treat neonatal abstinence syndrome: improved family-centered care at lower cost. Pediatrics. 2016;137(6):e20152929. https://doi.org/10.1542/peds.2015-2929.

9. Abrahams RR, Kelly SA, Payne S, Thiessen PN, Mackintosh J, Janssen PA. Rooming-in compared with standard care for newborns of mothers using methadone or heroin. Can Fam Physician. 2007;53(10):1722-30.

10. Hünseler C, Brückle M, Roth B, Kribs A. Neonatal opiate withdrawal and rooming-in: a retrospective analysis of a single center experience. Klin Padiatr. 2013;225(5):247-51. https://doi.org/10.1055/s-0033-1347190.

11. Saiki T, Lee S, Hannam S, Greenough A. Neonatal abstinence syndrome-postnatal ward versus neonatal unit management. Eur J Pediatr. 2010;169(1):95-8. https://doi.org/10.1007/s00431-009-0994-0.

12. Holmes AP, Schmidlin HN, Kurzum EN. Breastfeeding considerations for mothers of infants with neonatal abstinence syndrome. Pharmacotherapy. 2017;37(7):861-9. https://doi.org/10.1002/phar.1944.

13. Casper T, Arbour M. Evidence-based nurse-driven interventions for the care of newborns with neonatal abstinence syndrome. Adv Neonatal Care. 2014;14(6):376-80. https://doi.org/10.1097/ANC.0000000000 000118.

14. Kondili E, Duryea DG. The role of mother-infant bond in neonatal abstinence syndrome (NAS) management. Arch Psychiatr Nurs. 2019;33(3):267-74. https://doi.org/10.1016/j.apnu.2019.02.003.

15. Shuman CJ, Weber A, VanAntwerp K, Wilson R. Engaging mothers to implement nonpharmacological care for infants with neonatal abstinence syndrome: perceptions of perinatal and pediatric nurses. Adv Neonatal Care. 2020;20(6):464-72. https://doi.org/10.1097/ANC.00000 00000000812

16. Rockefeller K, Macken LC, Craig A. Trying to do what is best: a qualitative study of maternal-infant bonding and neonatal abstinence syndrome. Adv Neonatal Care. 2019;19(5):E3-E15. https://doi.org/10.1097/ANC. 0000000000000616

17. Maguire D, Webb M, Passmore D, Cline G. NICU nurses' lived experience: caring for infants with neonatal abstinence syndrome. Adv Neonatal Care. 2012;12(5):281-5. https://doi.org/10.1097/ANC.0b013e3182677bc1.

18. Atwood EC, Sollender G, Hsu E, Arsnow C, Flanagan V, Celenza J, et al. A qualitative study of family experience with hospitalization for neonatal abstinence syndrome. Hosp Pediatr. 2016;6(10):626-32. https://doi.org/ 10.1542/hpeds.2016-0024

19. Shuman CJ, Liu X, Aebersold ML, Tschannen D, Banaszak-Holl J, Titler MG. Associations among unit leadership and unit climates for implementation in acute care: a cross-sectional study. Implement Sci. 2018;13(1):62. https://doi.org/10.1186/s13012-018-0753-6.

20. Novick $\mathrm{G}$. Is there a bias against telephone interviews in qualitative research? Res Nurs Health. 2008;31(4):391-8. https://doi.org/10.1002/nur. 20259.

21. Sturges JE, Hanrahan KJ. Comparing telephone and face-to-face qualitative interviewing: a research note. Qual Res. 2004;4(1):107-18.
22. Trier-Bieniek A. Framing the telephone interview as a participant-centred tool for qualitative research: a methodological discussion. Qual Res. 2012;12(6):630-44

23. Cachia M, Millward L. The telephone medium and semi-structured interviews: a complementary fit. Qual Res Org Manage. 2011;6(3):265-77.

24. Damschroder LJ, Aron DC, Keith RE, Kirsh SR, Alexander JA, Lowery JC. Fostering implementation of health services research findings into practice: a consolidated framework for advancing implementation science. Implement Sci. 2009;4(1):50. https://doi.org/10.1186/1748-5908-4-50.

25. Glaser BG, Strauss AL. Discovery of grounded theory: strategies for qualitative research. New York: Routledge; 2017.

26. Jaye C. Doing qualitative research in general practice: methodological utility in engagement. Fam Pract. 2002;19(5):557-62.

27. Spehr MK, Coddington J, Ahmed AH, Jones E. Parental opioid abuse: barriers to care, policy, and implications for primary care pediatric providers. J Pediatr Health Care. 2017;31(6):695-702.

28. Warren Jl, McLaughlin M, Bardsley J, Eich J, Esche CA, Kropkowski L, et al. The strengths and challenges of implementing EBP in healthcare systems. Worldviews Evid-Based Nurs. 2016;13(1):15-24. https://doi.org/ 10.1111/wvn.12149.

29. Yoo JY, Kim JH, Kim JS, Kim HL, Ki JS. Clinical nurses' beliefs, knowledge, organizational readiness and level of implementation of evidence-based practice: The first step to creating an evidence-based practice culture. PLoS One. 2019;14(12):e0226742. https://doi.org/10.1371/journal.pone. 0226742.

30. Doede M, Trinkoff AM, Gurses AP. Neonatal intensive care unit layout and nurses' work. HERD. 2018;11(1):101-18. https://doi.org/10.1177/19375 86717713734.

31. Bosch S, Bledsoe T, Jenzarli A. Staff perceptions before and after adding single-family rooms in the NICU. HERD. 2012;5(4):64-75. https://doi.org/ $10.1177 / 193758671200500406$

32. O'Callaghan N, Dee A, Philip RK. Evidence-based design for neonatal units: A systematic review. Matern Health Neonatol Perinatol. 2019;5:6. https://doi.org/10.1186/s40748-019-0101-0.

33. Ramm K, Mannix T, Parry Y, Gaffney MP. A comparison of sound levels in open plan versus pods in a neonatal intensive care unit. HERD. 2017;10(3):30-9. https://doi.org/10.1177/1937586716668636.

34. Feeley N, Robins S, Genest C, Stremler R, Zelkowitz P, Charbonneau L. A comparative study of mothers of infants hospitalized in an open ward neonatal intensive care unit and a combined pod and single-family room design. BMC Pediatr. 2020;20(1):38. https://doi.org/10.1186/ s12887-020-1929-1.

\section{Publisher's Note}

Springer Nature remains neutral with regard to jurisdictional claims in published maps and institutional affiliations.
Ready to submit your research? Choose BMC and benefit from:

- fast, convenient online submission

- thorough peer review by experienced researchers in your field

- rapid publication on acceptance

- support for research data, including large and complex data types

- gold Open Access which fosters wider collaboration and increased citations

- maximum visibility for your research: over 100M website views per year

At BMC, research is always in progress.

Learn more biomedcentral.com/submissions 